European Organisation For Nuclear Research

CERN-EP/2001-025

6 March 2001

\title{
Benchmarking the Particle Background in the Large Hadron Collider Experiments
}

\author{
Edda Gschwendtner, Helmut Vincke, Christian W. Fabjan, \\ Nigel Hessey ${ }^{1}$, Thomas Otto
}

CERN, 1211 Geneva 23, Switzerland

\begin{abstract}
Background benchmarking measurements have been made to check the lowenergy processes which will contribute via nuclear reactions to the radiation background in the LHC experiments at CERN. Previously these processes were only evaluated with Monte Carlo simulations, estimated to be reliable within an uncertainty factor of 2.5. Measurements were carried out in an experimental set-up comparable to the shielding of ATLAS, one of the general-purpose experiments at LHC. The absolute yield and spectral measurements of photons and neutrons emanating from the final stages of the hadronic showers were made with a $\mathrm{Bi}_{4} \mathrm{Ge}_{3} \mathrm{O}_{12}$ (BGO) detector. The particle transport code FLUKA was used for detailed simulations. Comparison between measurements and simulations show that they agree within $20 \%$ and hence the uncertainty factor resulting from the shower processes can be reduced to a factor of 1.2 .
\end{abstract}

Submitted to Nuclear Instruments and Methods in Physics Research

\footnotetext{
${ }^{1}$ NIKHEF, Kruislaan 409, 1098 SJ Amsterdam, The Netherlands
} 


\section{Introduction}

The Large Hadron Collider (LHC) under construction at CERN is scheduled to be operational in 2006. The LHC will be a p-p collider with a centre-of-mass energy of $14 \mathrm{TeV}$. At a luminosity of $\mathcal{L}=10^{34} \mathrm{~cm}^{-2} \mathrm{~s}^{-1}$ the experiments will operate with collision rates at the $10^{9} \mathrm{~Hz}$ level, producing $>10^{11}$ particles per second. This very high rate of $\mathrm{p}-\mathrm{p}$ collisions creates a level of background so high that it becomes a major design criterion for the LHC experiments.

While the present work is relevant to all the LHC experiments, the detailed measurements were chosen to represent the ATLAS environment. ATLAS, A T(oroidal) L(HC) A(pparatu)S [1], is characterized by two distinct magnetic-field systems: A superconducting solenoid will be installed around the inner-detector cavity and large superconducting air-core toroids consisting of independent coils will be arranged outside the calorimetry for the muon spectrometer. This concept offers almost no constraints on calorimetry and the inner detector while at the same time providing magnetic fields for a high-resolution, large-acceptance and robust muon spectrometer.

\section{Particle background in the ATLAS environ- ment}

The ATLAS muon spectrometer defines the overall dimensions of the ATLAS detector. The barrel toroid extends over a length of $26 \mathrm{~m}$ with an inner diameter of $9.4 \mathrm{~m}$ and an outer diameter of $19.5 \mathrm{~m}$. The spectrometer is instrumented with high-precision tracking chambers that surround the calorimeter in three layers (radius 5-10 m).

The background emanating from the calorimeter and the shielding material is one of the main constraints on the muon spectrometer layout and operation. It influences parameters such as the rate performance of the readout electronics, the required radiation hardness of the instrumentation, the performance of the trigger system and the reconstruction quality.

Whereas the particles in the inner-detector cavity come mainly from the primary proton interactions with rates relatively well understood, the particle fluence in the muon spectrometer results from a chain of complex interactions in the calorimetry and shielding and is therefore affected by much larger uncertainties. Figure 1 shows the distribution of material shielding the muon system from primary-collision products.

Two broad classes of background contributions can be distinguished. First, there are primary-collision products such as hadronic debris of calorimeter showers 


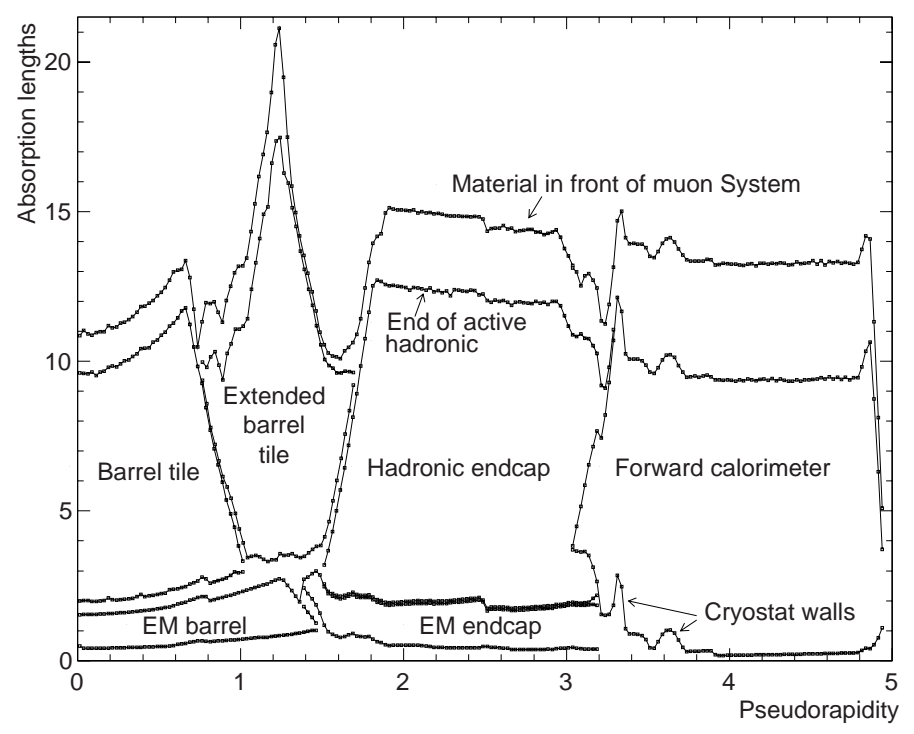

Figure 1: Contribution of the various ATLAS detectors to the absorptive thickness shielding the muon system from primary-collision products.

('punch-through'). The second and most important source of background are low-energy secondaries, mostly neutrons and photons, originating from primary hadrons interacting with the forward calorimeter, the shielding, the beam pipe and other machine elements. These low-energy neutrons will escape the absorber and create, via nuclear processes, a low-energy photon 'gas'.

Figure 2 shows the calculated photon fluence as a function of photon energy in different regions of the innermost region of the muon spectrometer behind the calorimeters as simulated by FLUKA [2].

The background rates have an uncertainty of a factor five (to be understood as a $2 \sigma$ level) owing to the superposition of different contributions: first, the uncertainties of the total $\mathrm{p}-\mathrm{p}$ cross-section and of the multiplicity produced in the primary collisions; second, the uncertainty of the muon-detector sensitivities; and lastly, and most importantly, the limited knowledge of the showering process in the absorber and of the (n, $\gamma)$ cross-sections and the ensuing neutron and photon production, which is estimated to contribute with a factor of 2.5.

This large uncertainty from the showering processes motivated the benchmarking measurements in an experimental set-up which is comparable to the ATLAS shielding. 


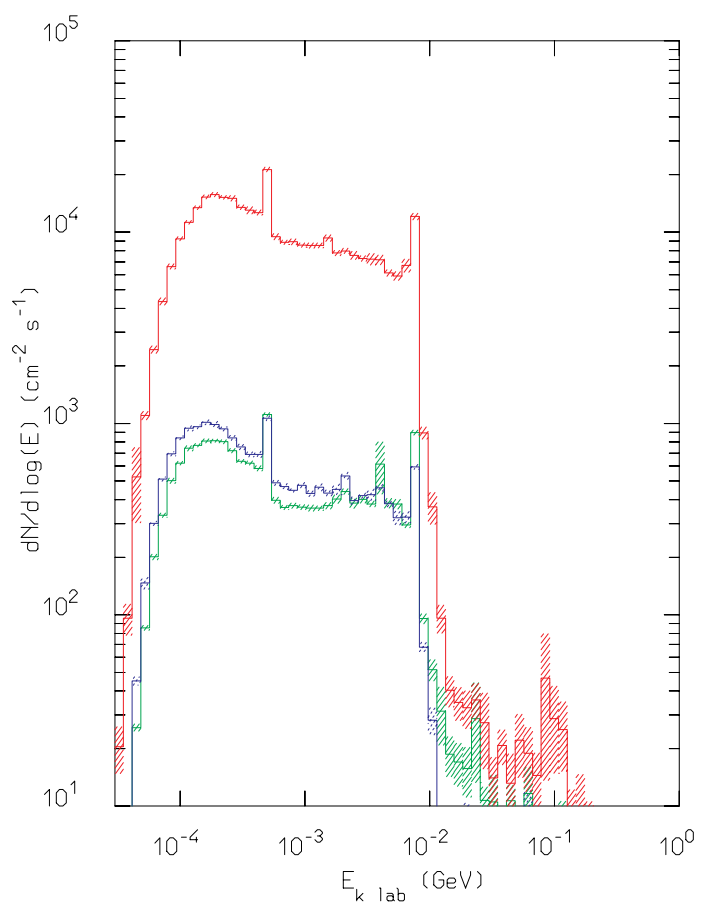

Figure 2: The expected photon flux as a function of photon energy in different regions of the muon spectrometer (top curve: $2.3<|\eta|<2.7$, middle curve: $1.4<|\eta|<2.3$ and bottom curve: $|\eta|<1.3)$.

\section{Benchmarking measurements}

\subsection{Experimental set-up}

A mixed, positively charged hadron beam $\left(\pi^{+}\right.$, protons, $\left.\mathrm{K}^{+}\right)$with a momentum of $p_{\text {beam }}=40 \mathrm{GeV} / c$ or $p_{\text {beam }}=120 \mathrm{GeV} / c$ was directed onto a $200 \mathrm{~cm} \times 200 \mathrm{~cm}$ cast-iron wall with a thickness of $200 \mathrm{~cm}(\approx 11 \lambda)$ and $240 \mathrm{~cm}(\approx 14 \lambda)$. This setup resembles closely the forward part (rapidity $\eta \geq 2$ ) of the ATLAS experiment, where the beam approximates the momenta of particles typically produced in 'minimum-bias collisions' and where the iron absorber corresponds to the shielding (i.e. calorimetry, beam-pipe, etc.) in front of the muon spectrometer. The set-up is shown in Fig. 3.

Since we were mainly interested in the photon background evaluation we have chosen a detector with a high photon detection efficiency. For this purpose a $\mathrm{Bi}_{4} \mathrm{Ge}_{3} \mathrm{O}_{12}$ (BGO) detector is very well suited since it is a high- $Z$ ( $Z=83$ for $\mathrm{Bi})$, high-density $\left(\rho=7.13 \mathrm{~g} / \mathrm{cm}^{3}\right)$ detector.

The cylindrical BGO detector (diameter and height $=3.8 \mathrm{~cm}$ ) was placed behind the iron block for the absolute rate and energy distribution measurements of particles, mostly photons and neutrons, emanating from the final stages of the 


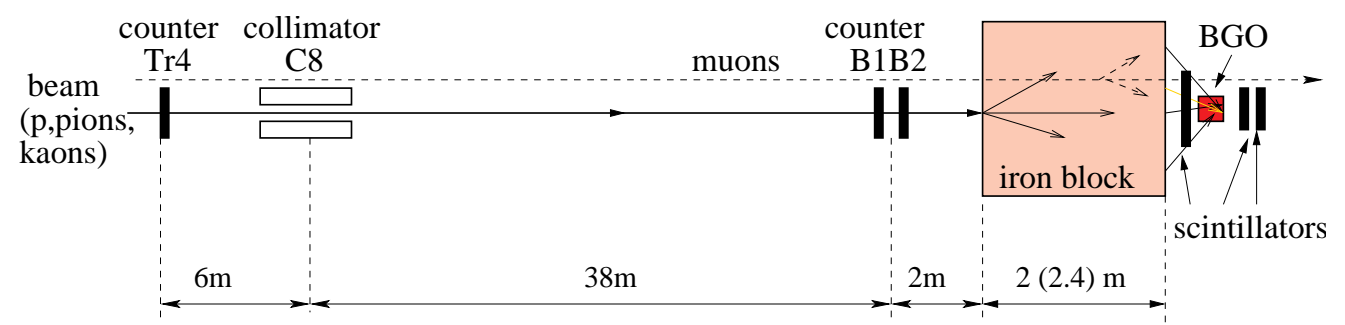

Figure 3: Sketch of the experimental area. Tr4 and B1B2 are the beam counters. The collimator $C 8$ can be closed in order to measure only the muon-induced signals. The distances are only approximate.

hadronic showers and induced from the beam hitting the absorber. In addition, three plastic scintillators were located between the iron wall and the BGO detector and downstream of the BGO detector. In different trigger arrangements, they were used to veto charged particles and muons for cross-check measurements.

Pion decays in the beam line and muons from neighbouring beam lines produce halo muons. These muons can initiate electromagnetic showers in the absorber, which in turn may produce a photon signal in the BGO detector.

Muon-induced photon signals were measured and subtracted by closing the beam collimator $\mathrm{C} 8$. In this configuration only muons reach the absorber allowing a measurement of this effect. In order to know the rate per beam particle the number of particles in the beam had to be measured. For that purpose we positioned two plastic scintillators B1 and B2 approximately $2 \mathrm{~m}$ in front of the iron absorber. We also recorded the scintillator Tr4 upstream of C8 in order to measure the number of beam particles with the collimator C8 closed.

\subsection{Discussion of the measurements}

Measurements were performed under a variety of conditions in order to achieve a good understanding of the experimental area, to reduce systematic effects, to assess residual gamma activities from other sources and to obtain data sets representative of different physics aspects of the hadronic cascade.

Hence measurements with different beam intensities $(\approx$ factor up to 3$)$ and beam momenta $\left(p_{\text {beam }}=40 \mathrm{GeV} / c\right.$ and $\left.p_{\text {beam }}=120 \mathrm{GeV} / c\right)$, different trigger conditions, different measurement positions $[\approx$ on beam-axis (on-axis) and $\approx 55 \mathrm{~cm}$ off beam-axis (off-axis)] and absorber thicknesses $(11 \lambda$ and $14 \lambda)$ were carried out. The studies give checks and corrections for spurious effects which could mask the measurements of the particle background.

For the different measurements the signal rate $R_{\mathrm{s}}$ of the particles that are induced by the beam particles impinging the iron absorber and emanating from 
the absorber is obtained by normalizing to the incoming beam rate

$$
R_{\mathrm{s}}=\frac{R_{d}}{R_{b}}
$$

The number of beam particles/s is $R_{b}$ and $R_{d}$ is the number of emanating particles/s induced by the beam particles and measured behind the absorber.

However, it is not possible to obtain these quantities in a single measurement. Several corrections have to be considered: as explained in Section 3.1 it is not possible to directly measure $R_{d}$ during a run, since the events that are measured by the BGO detector are not only induced by the beam particles, but also by the halo muons. Hence we have to subtract the muon-induced signals obtained by consecutive measurements with the collimator C8 opened and closed. Secondly, $R_{b}$ is measured with the scintillators $\operatorname{Tr} 4$ and B1B2, respectively. With the collimator $\mathrm{C} 8$ open, $\mathrm{B} 1 \mathrm{~B} 2$ gives $R_{b}$; with $\mathrm{C} 8$ closed $\mathrm{Tr} 4$ gives $R_{b}$. In addition, all counting rates have to be dead-time corrected and several offset values originating from the remanent radioactivity and other beam lines have to be measured and corrections applied.

Typically we expect to measure on average a photon energy deposit of $\approx 1 \mathrm{MeV}$ per $10^{3}$ incident hadrons, which is equivalent to a reduction factor in energy of $\approx 10^{8}$.

We find that the corrected and normalized particle rates $R_{\mathrm{s}}$ agree at a $1 \sigma$ level for the different intensity runs. We therefore conclude that the effects from residual activities, dead-time corrections and other experimental effects are well understood.

For the different measurements the uncertainties of corrections to the signal rates are within $6 \%$ and are dominated by the beam counter dead-time correction.

\section{Simulation}

\subsection{Characterization of the experimental environment}

For the simulation the version FLUKA 99 was used with the default 'precision'. In this version all the relevant physics effects are modelled, e.g. electrons, positrons and photons are transported down to $100 \mathrm{keV}$, neutrons are tracked down to $0.00001 \mathrm{eV}$.

The particle fluences at the on-axis and off-axis measurements are very different. On-axis particles are dominated by high-energy particle collisions with production of $\pi^{0}$ which decay into two $\gamma$ and initiate an electromagnetic shower. Therefore many $\gamma$ 's, electrons and positrons at late stages in the shower are observed. For 
such shower processes close to the end-face of the absorber and therefore close to the BGO detector, frequently more than one particle enters the detector at the same time. We therefore have a very high multi-event rate on-axis.

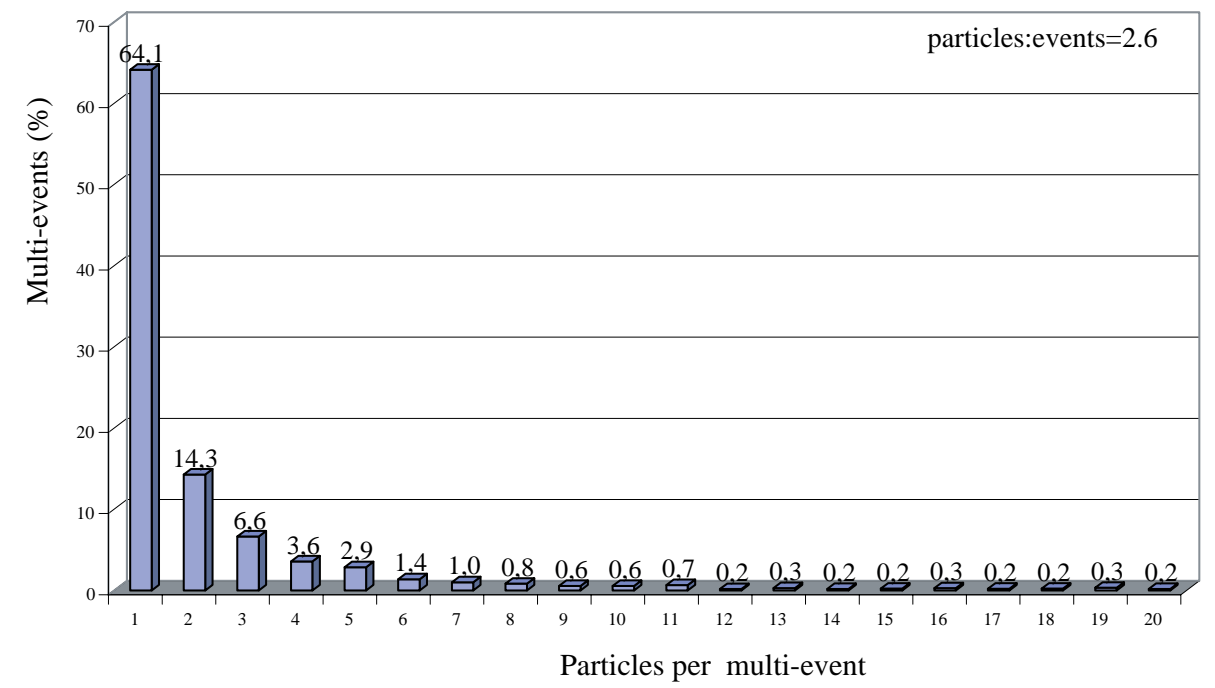

Figure 4: Classification of the multi-events behind $240 \mathrm{~cm}$ of iron, on-axis and with $p_{\text {beam }}=40 \mathrm{GeV} / \mathrm{c}$.

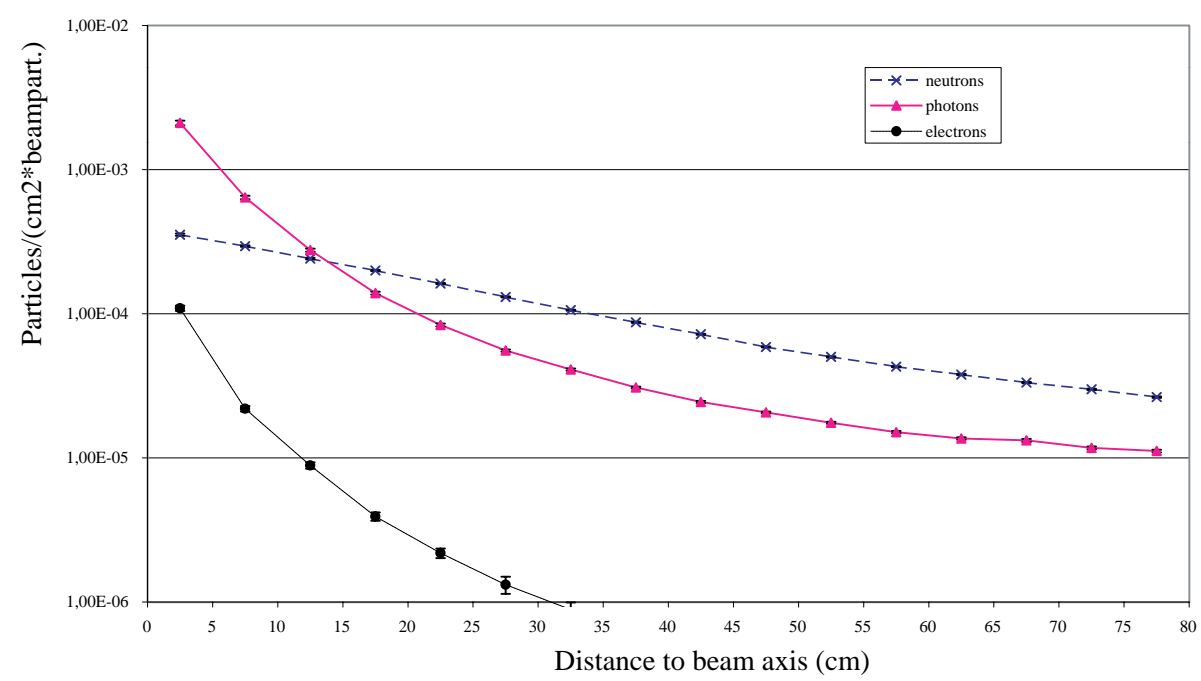

Figure 5: Particle fluences behind $240 \mathrm{~cm}$ of iron, and with $p_{\text {beam }}=40 \mathrm{GeV} / \mathrm{c}$.

In the measurements however, multi-events induced by such a shower event are registered as a single event. This fact has to be taken into account in the simulation. The multi-event composition for the $240 \mathrm{~cm}$ absorber and a beam momentum of $p_{\text {beam }}=40 \mathrm{GeV} / c$ on-axis is shown in Fig. 4 . Off-axis data are dominated by events where single particles enter the BGO detector. These particles are mainly neutrons and photons, which are produced by $(n, \gamma)$ and $\left(n, n^{\prime} \gamma\right)$ interactions in the absorber. The transverse distributions of fluences, again for $240 \mathrm{~cm}$ 
iron absorber and $p_{\text {beam }}=40 \mathrm{GeV} / c$ incident beam momentum, are shown in Fig. 5.

The procedure to simulate counting rates in the BGO detector was generally performed in three consecutive steps. In the first step the simulation of the shower processes induced in the absorber is calculated. Necessary information about particles hitting the BGO detector were subsequently used during a second run to compute the energy deposition spectrum in the detector crystal. In the third step this spectrum is convoluted with the energy-resolution function of the BGO detector.

In the first step two different methods are applied depending on the measurement position of the BGO detector. On-axis, the simulation has to be processed in 'analog mode'. Analog-mode simulations provide complete information of the correlation between the primary beam particles and the particles entering the BGO detector. By using this correlation the effect of the multi-events on the count rate is evaluated. At off-axis positions the fluence entering the BGO detector is very low. Therefore, 'biasing techniques' ('non-analog' simulation) can be used in order to increase the statistics of the results [2].

\section{Comparison of measurements with simulations}

\subsection{Measured and simulated signal rates}

Table 1 summarizes for all measurement conditions the total measured and simulated signal rates in the energy interval $0.35 \mathrm{MeV}<E<9 \mathrm{MeV}$ and also the normalized ratio. The measured rates are the averages of the two intensity runs per measurement position. All simulations were performed in the analog mode. In the simulation statistical and systematic uncertainties are included. The largest uncertainties are due to the imperfect knowledge of the BGO detector position and the carbon content in the iron.

The BGO detector position gives rise to the asymmetry in the uncertainty. Onaxis measurements with $200 \mathrm{~cm}$ absorber at $120 \mathrm{GeV} / c$ were not taken, as these conditions are not very representative of the LHC background conditions.

From the comparison we conclude that the measurements and the simulations agree very well. Most of the rates agree at a $20 \%$ level. The weighted average of all results yields a ratio of $1.13 \pm 0.06$. For the $200 \mathrm{~cm}$ iron absorber all simulations agree with the measurements within $1 \sigma$. The $240 \mathrm{~cm}$ absorber simulations are within $2 \sigma$. The uncertainties for the thick absorber $(240 \mathrm{~cm})$ are larger because of smaller statistics in the simulations. 
Table 1: Summary of measured and simulated signal rates in the energy interval $0.35 \mathrm{MeV}<E<9 \mathrm{MeV}$ per incident beam particle.

\begin{tabular}{|c|c|c|c|}
\hline \multirow{2}{*}{$\begin{array}{c}\text { Measurement } \\
\text { set-up }\end{array}$} & \multicolumn{2}{|c|}{ Signal rate $\times 10^{-4}$} & \multirow{2}{*}{$\begin{array}{c}\text { Ratio } \\
\text { meas./sim. }\end{array}$} \\
\hline & Measured & Simulated & \\
\hline \multicolumn{4}{|c|}{$\bar{p} p_{\text {beam }}=40 \mathrm{GeV} / c$} \\
\hline $200 \mathrm{~cm}$ on-axis & $76.2 \pm 0.4$ & $71.8_{-14.9}^{+6.7}$ & $1.06_{-0.22}^{+0.10}$ \\
\hline $200 \mathrm{~cm} \mathrm{o}$ & $6.8 \pm 0.2$ & $6.1_{-0.7}^{+0.8}$ & $1.12_{-0.13}^{+0.14}$ \\
\hline $240 \mathrm{~cm} \mathrm{o}$ & $23.0 \pm 0.4$ & $15.8 \pm 3.6$ & $1.46=0.33$ \\
\hline $240 \mathrm{~cm}$ off-axis & $2.3 \pm 0.1$ & $1.9 \pm 0.3$ & $1.24+0.15$ \\
\hline \multicolumn{4}{|c|}{$p_{\text {beam }}=120 \mathrm{GeV} / c$} \\
\hline $200 \mathrm{~cm}$ & $26.3 \pm 0.4$ & $26.0_{-2.9}^{+3.0}$ & $1.01+0.12$ \\
\hline $240 \mathrm{~cm} \mathrm{c}$ & $93.5 \pm 1.7$ & $78.6{ }_{-11.1}^{+9.6}$ & $1.19_{-0.17}^{+0.15}$ \\
\hline $240 \mathrm{~cm}$ off-axis & $9.7 \pm 0.6$ & $7.8_{-1.3}^{+1.4}$ & $1.24_{-0.21}^{+0.24}$ \\
\hline \multicolumn{3}{|c|}{ Weighted average } & $1.13 \pm 0.06$ \\
\hline
\end{tabular}

\subsection{Measured and simulated spectra}

In order to compare the measured and simulated spectra quantitatively we calculate the average energy deposition $\left\langle E_{\text {dep }}\right\rangle$ in the energy range $0.35 \mathrm{MeV}<E<$ $9 \mathrm{MeV}$ with

$$
\left\langle E_{\mathrm{dep}}\right\rangle=\frac{\sum_{i=E b i n_{\min }}^{E b i n_{\max }} R_{i} \cdot E_{i}}{R} \text { and } R=\sum_{i=E b i n_{\min }}^{E b i n_{\max }} R_{i}
$$

where $R_{i}$ is the normalized signal rate in the energy bin $i, E_{i}$ is the energy of the bin $i$ and $R$ is the total rate in the energy interval (see Table 1). The uncertainty on the energy deposition is smaller than the uncertainty on the rate since systematic uncertainties from dead-time corrections, imperfect BGO detector position knowledge, etc. have no or very little influence on the shape of the spectra. For the measurements the error is $\approx 1 \%$ and for the simulations it is $\approx 10 \%$.

Table 2 summarizes the averaged energy depositions. The measured and simulated results agree within the errors, except for $240 \mathrm{~cm}$, off-axis. Figure 6 shows the comparison between the measured and simulated spectra behind $240 \mathrm{~cm}$ of iron, on-axis and with $p_{\text {beam }}=120 \mathrm{GeV} / c$. At this position the neutron contribution is $\approx 10 \%$ of the total counting rate. The peak at $511 \mathrm{keV}$ originates from a pair production close to the BGO detector, where one annihilating photon enters the detector. We see that the shapes agree very well. Moreover, the average energy deposition shows very good agreement. Hence we can conclude that the complicated particle environment on-axis with the multi-events is well understood.

Figure 7 shows an example of the off-axis position. The results behind $200 \mathrm{~cm}$ 
Table 2: Summary of measured and simulated averaged energy depositions in the energy interval $0.35 \mathrm{MeV}<E<9 \mathrm{MeV}$.

\begin{tabular}{|c|c|c|}
\hline \multirow{2}{*}{$\begin{array}{l}\text { Measurement } \\
\text { set-up }\end{array}$} & \multicolumn{2}{|c|}{$\langle\overline{\langle E \text {-deposition }\rangle[\mathrm{MeV}]}$} \\
\hline & Measured & Simulated \\
\hline \multicolumn{3}{|c|}{$p_{\text {beam }}=40 \mathrm{GeV} / c$} \\
\hline $200 \mathrm{~cm}$ on-axis & $2.045 \pm 0.018$ & $2.057 \pm 0.174$ \\
\hline $200 \mathrm{~cm}$ off-axis & $1.615 \pm 0.004$ & $1.618 \pm 0.041$ \\
\hline $240 \mathrm{~cm}$ on-axis & $1.923 \pm 0.009$ & $1.824 \pm 0.073$ \\
\hline $240 \mathrm{~cm}$ off-axis & $1.666 \pm 0.003$ & $1.530 \pm 0.021$ \\
\hline \multicolumn{3}{|c|}{$p_{\text {beam }}=120 \mathrm{GeV} / c$} \\
\hline $200 \mathrm{~cm}$ off-axis & $1.572 \pm 0.009$ & $1.805 \pm 0.091$ \\
\hline $240 \mathrm{~cm}$ on-axis & $2.051 \pm 0.020$ & $2.058 \pm 0.183$ \\
\hline $240 \mathrm{~cm}$ off-axis & $1.649 \pm 0.006$ & $1.687 \pm 0.051$ \\
\hline
\end{tabular}

of iron absorber and $p_{\text {beam }}=40 \mathrm{GeV} / c$ can be seen. Off-axis approximately $30 \%$ of the counts are caused by pure neutron events, which is much higher than onaxis. In the simulation the neutron-induced counts add strong peaks to the entire spectrum. These peaks are not of a physical nature, but an artefact of FLUKA, arising from the discretization of the energy deposits of neutrons in the BGO detector. However, the fact that for both the simulations and the measurements the average energy deposition agrees better than 10\% shows that on average the energy deposits (both for photons and neutrons) in the BGO detector are simulated correctly.

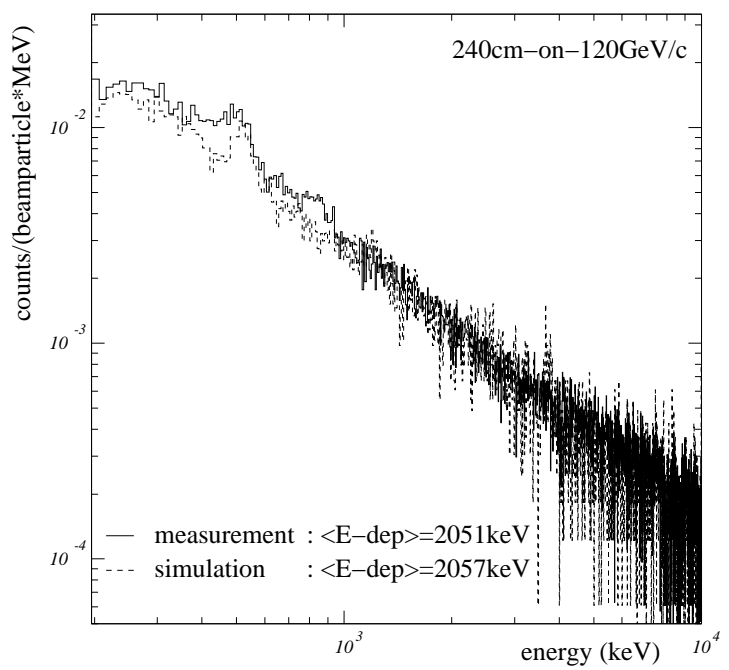

Figure 6: Measured and simulated total spectra behind $240 \mathrm{~cm}$ of iron, on-axis and $p_{\text {beam }}=120 \mathrm{GeV} / \mathrm{c}$. 


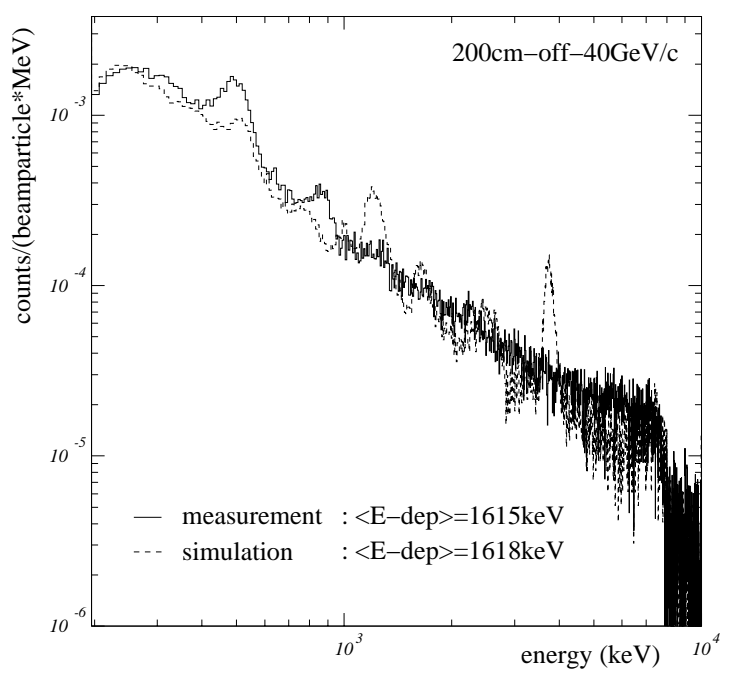

Figure 7: Measured and simulated total spectra behind $200 \mathrm{~cm}$ of iron, off-axis and $p_{\text {beam }}=40 \mathrm{GeV} / \mathrm{c}$.

\section{Conclusion}

Benchmarking measurements in a set-up comparable to the ATLAS shielding situation were performed in order to gauge the reliability of FLUKA simulations of the ATLAS background. Before these measurements, the background uncertainties due to the limited knowledge of the shower processes in the absorber and of the $(\mathrm{n}, \gamma)$ cross-sections were estimated to be at the 2.5 level. We have shown that the FLUKA simulations agree with the background benchmarking measurements to better than 20\%. As a consequence the uncertainties from the shower processes are now known within a factor of 1.2. Furthermore, our measurements provide a new yard-stick for the quality of FLUKA simulations in a regime which is dominated by detailed nuclear-physics processes. These benchmarks provide therefore a new level of reliability for a wealth of other applications.

\section{Acknowledgements}

We acknowledge the cooperation and help of the SL-EA group in setting up the beam line. A. Ferrari provided essential help through numerous discussions. 


\section{References}

[1] ATLAS, A General Purpose Experiment at LHC: ATLAS Technical Proposal, CERN/LHCC/94-43, LHCC/P2 (1994).

[2] A. Fasso et al., New Developments in FLUKA Modelling Hadronic and EM Interactions, Proc. 3rd Workshop on Simulating Accelerator Radiation Environments, KEK, Tsukuba, Japan 7-9 May 1997, Ed. H. Hirayama, KEK Proceedings 97-5, p. 32-43 (1997).

[3] H. Vincke, E. Gschwendtner, C.W. Fabjan, T. Otto, BGO Calibration Measurements and Simulations, to be submitted to Nucl. Instrum. Methods, March 2001.

[4] E. Gschwendtner, Benchmarking the Particle Background in the LHC Experiments, PhD thesis, CERN (2000).

[5] H. Vincke, Benchmarking of the ATLAS Hall Background Simulation, PhD thesis, CERN (2000). 